\title{
Almost Global Stability of Nonlinear Switched System with Stable and Unstable Subsystems
}

\author{
Ayşegül Kıvılcım, Özkan Karabacak and Rafael Wisniewski
}

\begin{abstract}
This paper presents sufficient conditions for almost global stability of nonlinear switched systems consisting of both stable and unstable subsystems. Techniques from the stability analysis of switched systems have been combined with the multiple Lyapunov density approach - recently proposed by the authors for the almost global stability of nonlinear switched systems composed of stable subsystems. By using slow switching for stable subsystems and fast switching for unstable subsystems lower and upper bounds for mode-dependent average dwell times are obtained. In addition to that, by allowing each subsystem to perform slow switching and using some restrictions on total operation time of unstable subsystems and stable subsystems, we have obtained a lower bound for an average dwell time.
\end{abstract}

\section{INTRODUCTION}

Global stability of switched systems has received the attention of researchers in the last two decades [1], [2], [3]. It has been widely investigated using multiple Lyapunov functions associated to each subsystem. Using decay rates of Lyapunov functions and the relations between the multiple Lyapunov functions, lower bounds for a minimum dwell time [1], [2] and an average dwell time [3] have been obtained for switched systems composed of only stable subsystems. Similarly, lower bounds for mode-dependent average dwell times [4] and edge-dependent average dwell times [5] have been obtained for these systems. It is natural to ask whether it is possible to stabilize a switched system if it comprises some unstable subsystems. The answer is affirmative. In [6], the authors have obtained a lower bound for an average dwell time to ensure global stability of the switched system. To this end, linear matrix inequalities have been employed. In [7], the authors have ensured stability by allowing stable subsystems to switch slow and unstable subsystem to switch fast. To ensure stability, they have utilized mode dependent average dwell times for switching signals. A mode-dependent average dwell time $\tau_{p}, p \in\{1,2, \ldots, M\}$ is an amount of time that the subsystem $p$ remains active on average over all of its activation periods [4]. As a result of that, they have obtained lower bounds for mode-dependent average dwell times of stable subsystems and upper bound for a mode-dependent average dwell time of unstable subsystems. Moreover, they have guaranteed stability by using a bound for the fraction

This work has been supported by the Independent Research Fund Denmark (DeBaTe).

A. Kivilcim and R. Wisniewski is with the Department of Electronic Systems, Aalborg University, 9200 Aalborg, Denmark ayk@es.aau.dk, rafees.aau.dk

Özkan Karabacak is with the Department of Electrical and Electronics Engineering, Bakırçay University, İzmir, Turkey oezkankarabacak@gmail.com of the total running time of all stable and the total running time of all unstable subsystems together with slow switching. Similarly, in [8], a lower bound for an average dwell time has been obtained with the help of the ratio between the operation time of stable and unstable subsystems, the decay and the growth rate of the multiple Lyapunov functions and the relations between multiple Lyapunov functions.

However, one cannot guarantee global stability for some systems that allow divergence of some solutions (of zero Lebesgue measure) to infinity. Nonetheless, one can guarantee almost global stability for such systems. In [9], Rantzer has proposed a tool called "Lyapunov density" to analyze almost global stability of autonomous systems. Subsequently, it has been extended to non-autonomous systems [10], systems with state dependent switching [11] and coupled systems [12]. In [13], we have generalized the main result in [9] to nonlinear switched systems with all modes stable. We have obtained a lower bound for a minimum dwell time. In [14], a lower bound for an average dwell time has been obtained for such systems. In contrast to those results, in this paper, we address a nonlinear switched system with stable and unstable subsystems.

Our motivation is to analyze almost global stability of the switched system using multiple Lyapunov densities. It can be seen as an extension of the results in [7]. We have obtained two sufficient conditions to ensure almost global stability with the help of transfer operators such as Koopman and Frobenius-Perron operators. Firstly, we derive lower bounds for mode-dependent average dwell times of stable subsystems and an upper bound for a mode-dependent average dwell time of unstable subsystems that guarantee almost global stability. Secondly, we obtain a lower bound for an average dwell time to ensure almost global stability. The lower bound relies on the ratio between the operation time of all stable and all unstable subsystems, the growth and the decay rate of densities along the solutions of subsystems, and the relation between densities.

The outline of the paper is as follows: Section II provides the preliminaries used in the paper and some lemmas used in proofs of the main theorems of the paper. Section III covers two sufficient conditions to ensure almost global stability of switched systems composed of stable and unstable subsystems. Section IV presents an example to show the applicability of the results in Section III.

Notation. $\mathbb{R}(\mathbb{Z}), \mathbb{R}_{>0}\left(\mathbb{Z}_{>0}\right)$ and $\mathbb{R}_{\geq 0}\left(\mathbb{Z}_{\geq 0}\right)$ denote the set of all, positive and non-negative real numbers (integers), respectively. $\mathbb{R}^{n}$ denotes the vector space of real $n$-tuples, $\|\cdot\|$ is used for the Euclidean norm. 0 denotes the zero 
vector in $\mathbb{R}^{n}$. For a map $f: \mathbb{R}^{n} \rightarrow \mathbb{R}^{n}, \nabla \cdot f$ stands for the divergence of $f$. For a function $g: \mathbb{R}^{n} \rightarrow \mathbb{R}, \nabla g$ denotes the gradient of $g$. The Lebesgue measure on $\mathbb{R}^{n}$ is denoted by $m$ and $\int \cdot d \mu(x)$ denotes Lebesgue integral with respect to a measure $\mu$. Specifically, Lebesgue integral with respect to Lebesgue measure $m$ will be denoted by $\int \cdot d x \equiv \int \cdot d m(x)$. $f: \mathbb{R}^{n} \rightarrow \mathbb{R}^{n}$ is said to be non-singular if $m\left(f^{-1}(A)\right)=0$ for every measurable set $A$ with $m(A)=0$. The phrases "almost all", "almost every" and "almost everywhere" will be used in the sense of Lebesgue measure. The characteristic function of a set $A$ is denoted by $1_{A}$. For an $\varepsilon>0$, let $B_{\varepsilon}:=\left\{x \in \mathbb{R}^{n} \mid\|x\|<\varepsilon\right\}$, and denote the complement of $B_{\varepsilon}$ as $B_{\varepsilon}^{c}$. We say that a function $f: \mathbb{R}^{n} \rightarrow \mathbb{R}$ is integrable away from $\mathbf{0}$ meaning that it is Lebesgue integrable on $B_{\varepsilon}^{c}$ for all $\varepsilon>0$.

\section{PRELIMINARIES}

In this part, we will provide some preliminaries and lemmas which will be used in the paper.

\section{A. Switched Systems}

This paper investigates a continuous-time nonlinear switched system of the following form

$$
\dot{x}(t)=f_{\sigma}(x(t)), \quad \sigma \in \mathcal{S},
$$

where $\sigma$ is the switching signal and $\mathcal{S}$ is the set of admissible switching signals which will be discussed later on. As the sets of admissible switching signals, we will consider the set of switching signals with average dwell time and mode dependent average dwell time properties.

The switching signal $\sigma:[0, \infty) \rightarrow \mathcal{P}, \mathcal{P}=\{1,2, \ldots, M\}$ is a right continuous and piece-wise constant function. Each system presented by $\dot{x}(t)=f_{p}(x(t)), p \in \mathcal{P}$, is called a subsystem of (1). In this paper, we assume that each subsystem $f_{p}: \mathbb{R}^{n} \rightarrow \mathbb{R}^{n}, p \in \mathcal{P}$, is continuously differentiable and $f_{p}(\mathbf{0})=\mathbf{0}, \forall p \in \mathcal{P}$. Let $t_{i}, i \in$ $\mathbb{Z}_{\geq 0}$, be the switching instants of the signal $\sigma$. Denote the value of the switching signal $\sigma(t)$ for $t \in\left[t_{i-1}, t_{i}\right)$ as $p_{i}$. For simplicity, we will represent the switching signal as $\sigma=\left\{\left(\Delta t_{1}, p_{1}\right),\left(\Delta t_{2}, p_{2}\right), \ldots\right\}$, where $\Delta t_{i}$ is the operation time of the subsystem $f_{p_{i}}$. Let subsystems with indices $\{1,2, \ldots, K\}$ be almost globally stable (see condition (12)) and $\{\mathrm{K}+1, \ldots, \mathrm{M}\}$ be unstable (see conditions (13) and (25)).

Definition 1: [13] The system (1) is said to be almost globally stable if for each $\sigma \in \mathcal{S}$, forward complete solutions $x: \mathbb{R}_{\geq 0} \rightarrow \mathbb{R}^{n}$ exist and converge to $\mathbf{0}$ for almost all initial states $x(0)=x_{0}$.

In the next section, we will analyze almost global stability of (1) with almost globally stable and unstable subsystems for switching signals satisfying average dwell time or modedependent average dwell time conditions. To this end, let us present the following definitions.

Definition 2: (Average dwell time)[3] For a switching signal $\sigma$ and any $t \geq 0$, denote the total number of switches during the time interval $[0, t)$ by $N(t)$. If there exist numbers $N_{0} \in \mathbb{Z}_{\geq 0}$ and $\tau \in \mathbb{R}_{>0}$, such that

$$
N(t) \leq N_{0}+\frac{t}{\tau}, \quad \forall t \geq 0
$$

we say that $\tau$ is an average dwell time and $N_{0}$ is a chatter bound of the switching signal $\sigma$. Denote the set of switching signals satisfying average dwell time condition (2) as $S_{A D T}[\tau]$.

Definition 3: (Mode-dependent average dwell times)[4] For a switching signal $\sigma$ and any $t \geq 0$, denote the total number of switches to the $p$-th subsystem during the time interval $[0, t)$ by $N_{p}(t)$ and denote the total amount of time when the subsystem $p$ is active during the time interval $[0, t)$ by $T_{p}(t)$, for $p \in \mathcal{P}$. If for all $p \in \mathcal{P}$ there exist numbers $N_{p}^{0} \in \mathbb{Z}_{\geq 0}$ and $\tau_{p} \in \mathbb{R}_{>0}$, such that

$$
N_{p}(t) \leq N_{p}^{0}+\frac{T_{p}(t)}{\tau_{p}}, \quad \forall t \geq 0, \forall p \in \mathcal{P},
$$

we say that $\left\{\tau_{p}\right\}_{p \in \mathcal{P}}$ is a set of mode-dependent average dwell times and $\left\{N_{p}^{0}\right\}_{p \in \mathcal{P}}$ is a set of mode-dependent chatter bounds of the switching signal $\sigma$.

Denote the set of switching signals satisfying mode-dependent average dwell time condition (3) as $S_{M D A D T}\left[\left\{\tau_{p}\right\}_{p \in \mathcal{P}}\right]$.

Definition 4: (A mode-dependent average dwell time for fast switching)[7] For a switching signal $\sigma$ and any $t \geq 0$, denote the total number of switches to the unstable subsystems during the time interval $[0, t)$ by $N^{\mathrm{f}}(t)$ and denote the total amount of time when the unstable subsystems are active during the time interval $[0, t)$ by $T^{\mathrm{f}}(t)$, for $p \in \mathcal{P}$. If there exist numbers $N^{\text {ff }} \in \mathbb{Z}_{\geq 0}$ and $\tau^{\mathrm{f}} \in \mathbb{R}_{>0}$, such that

$$
N^{\mathrm{f}}(t) \geq N^{0 \mathrm{f}}+\frac{T^{\mathrm{f}}(t)}{\tau^{\mathrm{f}}}, \quad \forall t \geq 0, \forall p \in \mathcal{P},
$$

we say that $\tau^{\mathrm{f}}$ is a mode-dependent average dwell time of the fast switching and $N^{0 f}$ is a mode-dependent chatter bound of the fast switching for the switching signal $\sigma$.

Denote the set of switching signals satisfying modedependent average dwell time condition for fast switching (4) as $S_{M D A D T}\left[\tau^{\mathrm{f}}\right]$.

\section{B. Transfer Operators: Perron and Koopman Operator}

The proofs of the main theorems lean upon transfer operators, Frobenius-Perron and Koopman operators [15]. These are defined on the set of equivalence classes of measurable functions, $\mathcal{M}\left(\mathbb{R}^{n} \backslash\{\mathbf{0}\}\right)$, where two functions are assumed to be equal if they are identical on a set with positive Lebesgue measure.

Assume that all solutions of $\dot{x}=f(x)$ exist and are unique for all forward times. Denote the time $t$ solution map of $\dot{x}=f(x)$ by $\phi_{t}(x)$, for each fixed $t \geq 0$ and $x \in \mathbb{R}^{n}$. Existence of unique solutions of $\dot{x}=f(x)$ for all forward times follows the non-singularity of the flow map $\phi_{t}$, for each fixed $t \geq 0$. Therefore, the Frobenius-Perron operator $P^{(t)}, t \geq 0$ is uniquely defined on $\mathcal{M}\left(\mathbb{R}^{n} \backslash\{\mathbf{0}\}\right)$ by

$$
\int_{A} P^{(t)} \rho(x) d x=\int_{\phi_{t}^{-1}(A)} \rho(x) d x
$$

for $\rho \in \mathcal{M}\left(\mathbb{R}^{n} \backslash\{\mathbf{0}\}\right)$, for any measurable set $A$ and for a fixed $t \geq 0$; here, $\phi_{t}^{-1}(A):=\left\{x \in \mathbb{R}^{n} \mid \phi_{t}(x) \in A\right\}$. Similarly, the Koopman operator $U^{(t)}$ is defined uniquely on $\mathcal{M}\left(\mathbb{R}^{n} \backslash\{\mathbf{0}\}\right)$ for each fixed $t \geq 0$ as

$$
U^{(t)} g(x)=g\left(\phi_{t}(x)\right),
$$


where $g \in \mathcal{M}\left(\mathbb{R}^{n} \backslash\{\mathbf{0}\}\right)$. Defining $\langle g, \rho\rangle:=\int_{\mathbb{R}^{n}} g \rho d x$, and verifying $\left\langle U^{(t)} g, \rho\right\rangle=\left\langle g, P^{(t)} \rho\right\rangle$, it can be shown that operators $P^{(t)}$ and $U^{(t)}$ are dual to each other for each fixed $t \geq 0$. For more details, see [15], [16] and the references therein. Note also that the transfer operators can be defined similarly if solutions exist for almost all initial states. This is because $\mathcal{M}\left(\mathbb{R}^{n} \backslash\{\mathbf{0}\}\right)$ is defined up to a set of measure zero [13].

\section{Transfer Operators for Switched Systems}

Denote for each fixed $\sigma \in \mathcal{S}$ and each fixed $t \geq 0$ and $x \in \mathbb{R}^{n}$, the time $t$ solution map of (1) by $\phi_{t}^{\sigma}(x)$. For each switching signal $\sigma \in \mathcal{S}$ and each fixed $t \geq 0$, FrobeniusPerron Operators, $\left\{P^{(t)}(\sigma)\right\}_{t \geq 0}$ and Koopman operators $\left\{U^{(t)}(\sigma)\right\}_{t \geq 0}$ can be defined respectively, as given in (5) and (6) for autonomous systems, with the help of the time $t$ solution map $\phi_{t}^{\sigma}(x)$ of (1) as follows:

$$
\begin{gathered}
\int_{A} P^{(t)}(\sigma) \rho(x) d x=\int_{\left(\phi_{t}^{\sigma}\right)^{-1}(A)} \rho(x) d x, \\
U^{(t)}(\sigma) g(x)=g\left(\phi_{t}^{\sigma}(x)\right),
\end{gathered}
$$

For $t \in\left[t_{n}, t_{n+1}\right), n \in \mathbb{Z}_{\geq 0}$, and let $t_{0}=0$, the FrobeniusPerron operator $P^{(t)}(\sigma)$ and the Koopman operator $U^{(t)}(\sigma)$ have the property that

$$
\begin{aligned}
& P^{(t)}(\sigma)=P_{p_{n+1}}^{\left(t-t_{n}\right)} P_{p_{n}}^{\left(t_{n}-t_{n-1}\right)} \cdots P_{p_{2}}^{\left(t_{2}-t_{1}\right)} P_{p_{1}}^{\left(t_{1}\right)} \\
& U^{(t)}(\sigma)=U_{p_{1}}^{\left(t_{1}\right)} U_{p_{2}}^{\left(t_{2}-t_{1}\right)} \cdots U_{p_{n}}^{\left(t_{n}-t_{n-1}\right)} U_{p_{n+1}}^{\left(t-t_{n}\right)}
\end{aligned}
$$

where $P_{p_{i}}^{(t)}$ and $U_{p_{i}}^{(t)}$ are the Frobenius-Perron and Koopman operators, respectively, corresponding to $p_{i}$-th active subsystem for the switching signal $\sigma$.

\section{Technical Lemmas}

The following lemmas are needed for the proofs of Theorem 1 and Theorem 2.

Lemma 1: (Slightly modified version of [13, Lemma 5]) Suppose that almost all solutions of $\dot{x}=f(x)$ exist for all $t \geq 0$, and $f: \mathbb{R}^{n} \rightarrow \mathbb{R}^{n}$ is continuously differentiable with $f(\mathbf{0})=\mathbf{0}$. Assume that there exist a constant $\kappa>0$ and a non-negative, continuously differentiable function $\rho$ : $\mathbb{R}^{n} \backslash\{\mathbf{0}\} \rightarrow \mathbb{R}_{\geq 0}$ such that $\rho(x)$ is integrable away from the origin and $\nabla \cdot(f \rho)(x) \geq \kappa \rho(x)$ for all $x \in \mathbb{R}^{n} \backslash\{\mathbf{0}\}$. Then, $P^{(t)} \rho(x) \leq \mathrm{e}^{-\kappa \mathrm{t}} \rho(x)$, for all $t>0$.

If $0>\nabla \cdot(f \rho)(x) \geq-\kappa \rho(x)$ for all $x \in \mathbb{R}^{n} \backslash\{\mathbf{0}\}$. Then, $P^{(t)} \rho(x) \leq \mathrm{e}^{\kappa \mathrm{t}} \rho(x)$, for all $t>0$.

Remark 1: The second part of Lemma 1 is used to show the evolution of density along the solution of an unstable system. It is an analogue of the increase of a Lyapunov-like function along solutions of an unstable subsystem [7]. In the main theorems, we will apply Lemma 1 to each stable and unstable subsystems separately to see the change of densities along the solutions of subsystems.

The following lemma can be seen as a Koopman operator characterization of almost global stability.

Lemma 2: [14] Assume that for each switching signal $\sigma \in$ $\mathcal{S}$, almost all solutions of the switched system (1) exist for all forward times. Then, (1) is almost globally stable if and only if $\int_{0}^{\infty} U^{(s)}(\sigma) 1_{B_{\varepsilon}^{c}}(x) d s<\infty$, for each $\sigma \in \mathcal{S}$ and almost every $x \in B_{\varepsilon}^{c}$ for all $\varepsilon>0$.

The following lemma is about almost global stability verification by using the Frobenius-Perron operators.

Lemma 3: [14] The switched system (1) is almost globally stable if for each $\sigma \in \mathcal{S}$, there exists an almost everywhere positive and measurable function $\rho$ on $\mathbb{R}^{n} \backslash\{\mathbf{0}\}$ such that $\int_{0}^{\infty} P^{(s)}(\sigma) \rho d s$ is integrable on $B_{\varepsilon}^{c}$ for all $\varepsilon>0$.

In the proofs of our theorems, we will ensure almost global stability of a switched system composed of stable and unstable subsystems leaning on Lemma 3.

\section{MAin Results}

Motivated by the papers [6], [7], [8], we present two sufficient conditions to ensure almost global stability of a switched system with stable and unstable subsystems.

The next theorem provides a sufficient condition to ensure almost global stability of the switched system by using a slow switching for the stable subsystems and a fast switching for the unstable subsystems. It can be seen as a generalization of the results obtained in [7] to almost global stability.

Theorem 1: Consider the switched system (1) with $M$ subsystems. Suppose that $K<M$ and there exist constants $\kappa>0, \kappa_{p}>0, p=1,2, \ldots, K$ and non-negative continuously differentiable functions $\rho: \mathbb{R}^{n} \backslash\{\mathbf{0}\} \rightarrow \mathbb{R}$ and $\rho_{p}: \mathbb{R}^{n} \backslash\{\mathbf{0}\} \rightarrow \mathbb{R}, p=1,2, \ldots, K$ such that

$\left(1+\left\|f_{p}\right\|\right) \rho_{p}(x)$ is integrable away from $\mathbf{0}, \forall p \in\{1, \ldots, K\} \&$

$\left(1+\left\|f_{p}\right\|\right) \rho(x)$ is integrable away from $0, \forall p \in\{K+1, \ldots, M\}(11)$

$\nabla \cdot\left(\rho_{p} f_{p}\right)(x) \geq \kappa_{p} \rho_{p}(x), \forall x \in \mathbb{R}^{n} \backslash\{\mathbf{0}\}, \forall p \in\{1, \ldots, K\}$, (12)

$0>\nabla \cdot\left(\rho f_{p}\right)(x) \geq-\kappa \rho(x), \forall x \in \mathbb{R}^{n} \backslash\{\mathbf{0}\}, \forall p \in\{K+1, \ldots, M\}(13)$

Suppose also that there exist positive numbers $c_{p} p \in$ $\{1,2 \ldots, K\}$ and $0<c<1$ such that

$$
\begin{aligned}
& \rho_{p}(x) \leq c_{p} \rho_{q}(x), \forall x \in \mathbb{R}^{n} \backslash\{\mathbf{0}\}, p, q \in\{1, \ldots, K\}, \\
& \rho_{p}(x) \leq c_{p} \rho(x), \forall x \in \mathbb{R}^{n} \backslash\{\mathbf{0}\}, p \in\{1, \ldots, K\}, \\
& \rho(x) \leq c \rho_{p}(x), \forall x \in \mathbb{R}^{n} \backslash\{\mathbf{0}\}, p \in\{1, \ldots, K\}
\end{aligned}
$$

Then, (1) with $\mathcal{S}=\mathcal{S}_{M D A D T}\left[\left\{\tau_{p}\right\}_{p \in\{1, \ldots, K\}}\right] \cup$ $S_{M D A D T}\left[\tau^{\mathrm{f}}\right]$, (the set of switching signals satisfying the slow switching mode-dependent average dwell time condition (3) and the fast switching mode-dependent average dwell time condition (4)) is almost globally stable if

$$
\tau_{p}>\tau_{p}^{\mathrm{ave}}:=\frac{\ln \left(c_{p}\right)}{\kappa_{p}}, p \in\{1,2, \ldots, K\}
$$

and

$$
\tau^{\mathrm{f}}<\tau^{\text {ave }}:=-\frac{\ln (c)}{\kappa} .
$$

Proof: Take an arbitrary switching signal $\sigma \in$ $\mathcal{S}_{M D A D T}\left[\left\{\tau_{p}\right\}_{p \in\{1, \ldots, K\}}\right] \cup S_{M D A D T}\left[\tau^{\mathrm{f}}\right]$. Let $t \in\left[t_{i}, t_{i+1}\right)$, where $t_{i}, i \in \mathbb{Z}_{\geq 0}$ are the switching instants of the signal $\sigma$ and for simplicity assume that $t_{0}=0$. The proof is complete from Lemma 3 if we show that $\int_{0}^{\infty} P^{(s)}(\sigma) \rho_{p_{1}} d s$ is integrable on $B_{\varepsilon}^{c}$, for all $\varepsilon>0$. Condition (11) implies almost global existence of solutions of (1), for more details see [13]. Condition (11) implies that $\rho_{p}, p \in\{1, \ldots, K\}$ 
and $\rho$ are integrable on $B_{\varepsilon}^{c}$, for all $\varepsilon>0$. For simplicity, we will assume that $\kappa_{K+1}=\ldots=\kappa_{M}=-\kappa$, and $c_{p}:=c$ if $p \in\{K+1, \ldots, M\}$. Using (9) and Lemma 1 with (12) and (13), we get

$$
\begin{aligned}
& P^{(t)}(\sigma) \rho_{p_{1}}=P_{p_{n+1}}^{\left(t-t_{n}\right)} P_{p_{n}}^{\left(t_{n}-t_{n-1}\right)} \ldots P_{p_{1}}^{\left(t_{1}\right)} \rho_{p_{1}} \\
& \leq \rho_{\max } \cdot \mathrm{e}^{-\kappa_{\mathrm{p}_{\mathrm{n}+1}}\left(\mathrm{t}-\mathrm{t}_{\mathrm{n}}\right)+\sum_{\mathrm{i}=1}^{\mathrm{n}}-\kappa_{\mathrm{p}_{\mathrm{i}}}\left(\mathrm{t}_{\mathrm{i}}-\mathrm{t}_{\mathrm{i}-1}\right)} \prod_{\mathrm{i}=1}^{\mathrm{n}} \mathrm{c}_{\mathrm{p}_{\mathrm{i}}} \\
& \leq \rho_{\max } \cdot \mathrm{e}^{-\kappa_{\mathrm{p}_{\mathrm{n}+1}}\left(\mathrm{t}-\mathrm{t}_{\mathrm{n}}\right)+\sum_{\mathrm{i}=1}^{\mathrm{n}}-\kappa_{\mathrm{p}_{\mathrm{i}}}\left(\mathrm{t}_{\mathrm{i}}-\mathrm{t}_{\mathrm{i}-1}\right)+\ln \left(\mathrm{c}_{\mathrm{p}_{\mathrm{i}}}\right)},
\end{aligned}
$$

where $\rho_{\max }:=\max \left\{\max _{p \in\{1, \ldots, K\}} \rho_{p}(x), \rho(x)\right\}, x \in \mathbb{R}^{n} \backslash\{\mathbf{0}\}$. Considering the switching instants of $\sigma$, we have

$$
\int_{0}^{\infty} P^{(s)}(\sigma) \rho_{p_{1}} d s=\sum_{n=0}^{\infty} \int_{t_{n}}^{t_{n+1}} P^{(s)}(\sigma) \rho_{p_{1}} d s .
$$

Using (19), (20) can be bounded as

$$
\begin{aligned}
& \int_{0}^{\infty} P^{(s)}(\sigma) \rho_{p_{1}} d s=\sum_{n=0}^{\infty} \int_{t_{n}}^{t_{n+1}} P^{(s)}(\sigma) \rho_{p_{1}} d s \\
& \leq \rho_{\max } \sum_{n=0}^{\infty} \int_{t_{n}}^{t_{n+1}}\left(\mathrm{e}^{-\kappa_{\mathrm{p}_{\mathrm{n}+1}}\left(\mathrm{~s}-\mathrm{t}_{\mathrm{n}}\right)+\sum_{\mathrm{i}=1}^{\mathrm{n}}-\kappa_{\mathrm{p}_{\mathrm{i}}}\left(\mathrm{t}_{\mathrm{i}}-\mathrm{t}_{\mathrm{i}-1}\right)+\ln \left(\mathrm{c}_{\mathrm{p}_{\mathrm{i}}}\right)}\right) \mathrm{ds} \\
& =\rho_{\max }\left(\frac{\mathrm{e}^{-\kappa_{p_{1}} t_{1}}-1}{-\kappa_{p_{1}}}+\sum_{n=1}^{\infty}\left(\mathrm{e}^{\sum_{\mathrm{i}=1}^{\mathrm{n}}\left(-\kappa_{\mathrm{p}_{\mathrm{i}}}\left(\mathrm{t}_{\mathrm{i}}-\mathrm{t}_{\mathrm{i}-1}\right)+\ln \left(\mathrm{c}_{\mathrm{p}_{\mathrm{i}}}\right)\right)} \times\right.\right. \\
& \left.\left.\times \frac{\mathrm{e}^{-\kappa_{\mathrm{p}_{\mathrm{n}+1}}\left(\mathrm{t}_{\mathrm{n}+1}-\mathrm{t}_{\mathrm{n}}\right)}-1}{-\kappa_{p_{n+1}}}\right)\right) \text {. }
\end{aligned}
$$

Note that $\kappa_{p_{n}}=\kappa_{p}, p_{n} \in\{1,2, \ldots, K\}$ or $\kappa_{p_{n}}=-\kappa$, $p_{n} \in\{K+1, K+2, \ldots, M\}$. Moreover, (4) implies that $t_{i+1}-t_{i} \leq T$, for some $T>0$ if $p_{i} \in\{k+1, \ldots, M\}$. It follows that $\frac{1-\mathrm{e}^{-\kappa_{\mathrm{p}_{\mathrm{n}+1}}\left(\mathrm{t}_{\mathrm{n}+1}-\mathrm{t}_{\mathrm{n}}\right)}}{\kappa_{p_{n+1}}} \leq M_{1}$, where $M_{1}=$ $\max \left\{\max _{p \in\{1, \ldots, K\}} \frac{1}{\kappa_{p}}, \frac{\mathrm{e}^{\kappa \mathrm{T}}}{\kappa}\right\}$. Then, the integral in (21) can be bounded as follows

$$
\int_{0}^{\infty} P^{(s)}(\sigma) \rho_{p_{1}} d s \leq M_{1} \rho_{\max }\left(1+\sum_{n=1}^{\infty}\left(\sum^{\mathrm{n}=1}\left(-\kappa_{\mathrm{p}_{\mathrm{i}}}\left(\mathrm{t}_{\mathrm{i}}-\mathrm{t}_{\mathrm{i}-1}\right)+\ln \left(\mathrm{c}_{\mathrm{p}_{\mathrm{i}}}\right)\right)\right)\right) \text {. }
$$

Considering $T_{p}(t)$ and $N_{p}(t)$ in Definition 3 and $T^{\mathrm{f}}(t)$ and $N^{\mathrm{f}}(t)$ in Definition 4 with $t:=t_{n}$, define $\bar{T}_{p}(n):=T_{p}\left(t_{n}\right)$, $\bar{N}_{p}(n):=N_{p}\left(t_{n}\right), \bar{T}^{\mathrm{f}}(n):=T^{\mathrm{f}}\left(t_{n}\right)$, and $\bar{N}^{\mathrm{f}}(n):=N^{\mathrm{f}}\left(t_{n}\right)$. Considering $\bar{T}_{p}(n), \bar{N}_{p}(n), \bar{T}^{\mathrm{f}}(n)$ and $\bar{N}^{\mathrm{f}}(n)$, instead of switching instant in (22), we have

$$
\begin{aligned}
& \int_{0}^{\infty} P^{(s)}(\sigma) \rho_{p_{1}} d s \leq M_{1} \rho_{\max }\left(1+\sum_{n=1}^{\infty}\left(e^{\sum^{p=1}}\left(-\kappa_{p} \bar{T}_{p}(n)+\ln \left(c_{p}\right) \bar{N}_{p}(n)\right)\right.\right. \\
& \left.\times \mathrm{e}^{\left(\kappa \overline{\mathrm{T}}^{\mathrm{f}}(\mathrm{n})+\ln (\mathrm{c}) \overline{\mathrm{N}}^{\mathrm{f}}(\mathrm{n})\right)}\right) .
\end{aligned}
$$

Note that (3) with $t=t_{n}$ implies that $\bar{T}_{p}(n) \geq\left(\bar{N}_{p}(n)-\right.$ $\left.N_{p}^{0}\right) \tau_{p}, p \in\{1,2, \ldots, K\}$. Similarly, (4) follows that $\bar{T}^{\mathrm{f}}(n) \leq\left(\bar{N}^{\mathrm{f}}(n)-N_{p}^{0}\right) \tau^{\mathrm{f}}$. Applying these to (23), we obtain

$$
\begin{aligned}
& \int_{0}^{\infty} P^{(s)}(\sigma) \rho_{p_{1}} d s \leq M_{1} \rho_{\max }(1+ \\
& \left.+\sum_{n=1}^{\infty} \mathrm{e}^{\sum_{\mathrm{p}=1}^{\mathrm{K}}\left(-\kappa_{\mathrm{p}}\left(\overline{\mathrm{N}}_{\mathrm{p}}(\mathrm{n})-\mathrm{N}_{\mathrm{p}}^{0}\right) \tau_{\mathrm{p}}+\ln \left(\mathrm{c}_{\mathrm{p}}\right) \overline{\mathrm{N}}_{\mathrm{p}}(\mathrm{n})\right)+\left(\kappa\left(\overline{\mathrm{N}}^{\mathrm{f}}(\mathrm{n})-\mathrm{N}^{0 \mathrm{f}}\right) \tau^{\mathrm{f}}+\ln (\mathrm{c}) \overline{\mathrm{N}}^{\mathrm{f}}(\mathrm{n})\right)}\right) \\
& =M_{1} \rho_{\max }\left(1+\sum_{n=1}^{\infty}\left(\mathrm{e}^{\sum_{\mathrm{p}=1}^{\mathrm{K}}\left(-\kappa_{\mathrm{p}}\left(\overline{\mathrm{N}}_{\mathrm{p}}(\mathrm{n})-\mathrm{N}_{\mathrm{p}}^{0}\right) \tau_{\mathrm{p}}+\ln \left(\mathrm{c}_{\mathrm{p}}\right) \overline{\mathrm{N}}_{\mathrm{p}}(\mathrm{n})\right)} \times\right.\right. \\
& \left.\times \mathrm{e}^{\left(\kappa\left(\overline{\mathrm{N}}^{\mathrm{f}}(\mathrm{n})-\mathrm{N}^{\mathrm{Of}}\right) \tau^{\mathrm{f}}+\ln (\mathrm{c}) \overline{\mathrm{N}}^{\mathrm{f}}(\mathrm{n})\right)}\right), \\
& =M_{1} \rho_{\max }\left(1+\mathrm{e}^{\sum_{\mathrm{p}=1}^{\mathrm{K}}\left(\kappa_{\mathrm{p}} \mathrm{N}_{\mathrm{p}}^{\mathrm{O}} \tau_{\mathrm{p}}\right)+\left(-\kappa \mathrm{N}^{\mathrm{Of}} \tau^{\mathrm{f}}\right)} \times\right. \\
& \times \sum_{n=1}^{\infty}\left(\mathrm{e}^{\sum_{\mathrm{p}=1}^{\mathrm{K}}\left(\left(-\kappa_{\mathrm{p}} \tau_{\mathrm{p}}+\ln \left(\mathrm{c}_{\mathrm{p}}\right)\right) \overline{\mathrm{N}}_{\mathrm{p}}(\mathrm{n})\right)} \mathrm{e}^{\left(\left(\kappa \tau^{\mathrm{f}}+\ln (\mathrm{c})\right) \overline{\mathrm{N}}^{\mathrm{f}}(\mathrm{n})\right)}\right),
\end{aligned}
$$

Note that if $n$ increases by 1 either $\bar{N}_{p}(n)$ or $\bar{N}^{\mathrm{f}}(n)$ increase by 1 . Let $\gamma:=\max \left\{\max _{p \in\{1, \ldots, K\}}\left(-\kappa_{p} \tau_{p}+\ln \left(c_{p}\right)\right),\left(\kappa \tau^{\mathrm{f}}+\right.\right.$ $\ln (c))\}$. Conditions (17) and (18) imply that $\gamma<0$. Then, above integral can be bounded as

$$
\begin{aligned}
& \int_{0}^{\infty} P^{(s)}(\sigma) \rho_{p_{1}} d s \leq M_{1} \rho_{\max }\left(1+\mathrm{e}^{\left(\sum_{\mathrm{p}=1}^{\mathrm{K}}\left(\kappa_{\mathrm{p}} \mathrm{N}_{\mathrm{p}}^{0} \tau_{\mathrm{p}}\right)\right)+\left(-\kappa \mathrm{N}^{\mathrm{Of}} \tau^{\mathrm{f}}\right)} \sum_{\mathrm{n}=1}^{\infty} \mathrm{e}^{\gamma \cdot \mathrm{n}}\right) \\
& \leq M_{1} \rho_{\max } \cdot\left(1+\mathrm{e}^{\left(\sum_{\mathrm{p}=1}^{\mathrm{K}}\left(\kappa_{\mathrm{p}} \mathrm{N}_{\mathrm{p}}^{0} \tau_{\mathrm{p}}\right)\right)-\left(\kappa \mathrm{N}^{\mathrm{Of}} \tau^{\mathrm{f}}\right)}\left(\frac{\mathrm{e}^{\gamma}}{1-\mathrm{e}^{\gamma}}\right)\right) .
\end{aligned}
$$

As a result $\int_{0}^{\infty} P^{(s)}(\sigma) \rho_{p_{1}} d s<\infty$, we can conclude by using Lemma 3 that (1) with $\mathcal{S}=\mathcal{S}_{M D A D T}\left[\left\{\tau_{p}\right\}_{p \in\{1, \ldots, K\}}\right] \cup$ $S_{M D A D T}\left[\tau^{\mathrm{f}}\right]$ is almost globally stable if conditions (17) and (18) are satisfied.

Remark 2: Condition (12) is used to constrain the change of densities along the solutions of stable subsystems. Likewise, condition (13) is used to constrain the change of density along the solutions of unstable subsystems. The conditions (14)-(16) represent the relations between multiple Lyapunov densities. Note that the density is taken as common for all unstable subsystems. If we associate a multiple Lyapunov density to each unstable subsystem, we should consider in each transition $c_{p}<1, p \in\{K+1, \ldots, M\}$, i.e., $\rho_{p} \leq c_{p} \rho_{q}$, where $p, q \in\{K+1, \ldots, M\}$. If we switched back form the subsystem $q$ to the subsystem $p$, we get $\rho_{q} \leq c_{q} \rho_{p}$. Then, together with previous inequality, we get $\rho_{p} \leq c_{p} c_{q} \rho_{p}$ that is not valid since $c_{p} c_{q}<1$.

Almost global stability of (1) can still be guaranteed without assuming fast switching for unstable subsystems. In the sequel, we will present a sufficient condition to ensure almost global stability by allowing slow switching to unstable subsystems as motivated by [6] and [8].

Theorem 2: Consider system (1). Assume that for each $p \in \mathcal{P}$, there exist constants $\kappa_{p}>0$, and non-negative continuously differentiable function $\rho_{p}: \mathbb{R}^{n} \backslash\{\mathbf{0}\} \rightarrow \mathbb{R}$ 
satisfying condition (12) and

$\left(1+\left\|f_{p}\right\|\right) \rho_{p}(x)$ are integrable away from $\mathbf{0}, \forall p \in \mathcal{P}$ (24)

$0>\nabla \cdot\left(\rho_{p} f_{p}\right)(x) \geq-\kappa_{p} \rho_{p}(x), \forall x \in \mathbb{R}^{n} \backslash\{\mathbf{0}\}$,

$p \in\{K+1, \ldots, M\}$.

Suppose also that there exist numbers $c_{p q}, p, q \in \mathcal{P}$ such that

$$
\rho_{p} \leq c_{p q} \rho_{q}, p, q \in \mathcal{P} \text {. }
$$

Then, (1) with $\mathcal{S}=\mathcal{S}_{A D T}[\tau]$ is almost globally stable if the following conditions are satisfied

$$
\begin{aligned}
& \frac{T^{-}(t)}{T^{+}(t)}>\frac{\kappa^{+}+\kappa^{*}}{\kappa^{-}-\kappa^{*}}, \quad \forall t>0 \\
& \tau>\max _{p, q \in \mathcal{P}} \frac{\ln \left(c_{p q}\right)}{\kappa^{*}},
\end{aligned}
$$

where $T^{-}(t)$ is the total running time of stable subsystems during the time interval $[0, t), T^{+}(t)$ is the total running time of unstable subsystems during the time interval $[0, t), \kappa^{-}=$ $\min _{p \in\{1, \ldots, K\}} \kappa_{p}, \kappa^{+}=\max _{p \in\{K+1, \ldots, M\}} \kappa_{p}$ and $\kappa^{*} \in\left(0, \kappa^{-}\right)$.

Proof: Take $\sigma \in \mathcal{S}_{A D T}[\tau]$. Let $t \in\left[t_{i}, t_{i+1}\right)$, where $t_{i}$, $i \in \mathbb{Z}_{>0}$ is the $i-$ th switching instant of $\sigma$ and assume that $t_{0}=0$. Condition (24) implies that $\rho_{p}, p \in \mathcal{P}$ are integrable on $B_{\varepsilon}^{c}, \forall \varepsilon>0$. Utilizing (9) and Lemma 1 with (12) and (25), we have

$$
\begin{aligned}
& P^{(t)}(\sigma) \rho_{p_{1}}=P_{p_{n+1}}^{\left(t-t_{n}\right)} P_{p_{n}}^{\left(t_{n}-t_{n-1}\right)} \ldots P_{p_{1}}^{\left(t_{1}\right)} \rho_{p_{1}} \\
& \leq \rho_{\max } \cdot \mathrm{e}^{-\kappa_{\mathrm{p}_{\mathrm{n}+1}}\left(\mathrm{t}-\mathrm{t}_{\mathrm{n}}\right)+\sum_{\mathrm{i}=1}^{\mathrm{n}}-\kappa_{\mathrm{p}_{\mathrm{i}}}\left(\mathrm{t}_{\mathrm{i}}-\mathrm{t}_{\mathrm{i}-1}\right)+\ln \left(\mathrm{c}_{\mathrm{p}_{\mathrm{i}}} \mathrm{p}_{\mathrm{i}+1}\right)},
\end{aligned}
$$

where $\rho_{\max }:=\max _{p \in \mathcal{P}} \rho_{p}(x), x \in \mathbb{R}^{n} \backslash\{\mathbf{0}\}$. Recall (20) in the proof of Theorem 1. Using (29), (20) can be evaluated as $\int_{0}^{\infty} P^{(s)}(\sigma) \rho_{p_{1}} d s=\sum_{n=0}^{\infty} \int_{t_{n}}^{t_{n+1}} P^{(s)}(\sigma) \rho_{p_{1}} d s$
$\leq \rho_{\max } \sum_{n=0}^{\infty} \int_{t_{n}}^{t_{n+1}}\left(\mathrm{e}^{-\kappa_{\mathrm{p}_{\mathrm{n}+1}}\left(\mathrm{~s}-\mathrm{t}_{\mathrm{n}}\right)+\sum_{\mathrm{i}=1}^{\mathrm{n}}-\kappa_{\mathrm{p}_{\mathrm{i}}}\left(\mathrm{t}_{\mathrm{i}}-\mathrm{t}_{\mathrm{i}-1}\right)+\ln \left(\mathrm{c}_{\left.\mathrm{p}_{\mathrm{i}} \mathrm{c}_{\mathrm{p}_{\mathrm{i}}+1}\right)}\right)}\right) \mathrm{ds}$ $=\rho_{\max }\left(\frac{\mathrm{e}^{-\kappa_{p_{1}} t_{1}}-1}{-\kappa_{p_{1}}}+\sum_{n=1}^{\infty}\left(\mathrm{e}^{\sum^{\mathrm{i}=1}\left(-\kappa_{\mathrm{p}_{\mathrm{i}}}\left(\mathrm{t}_{\mathrm{i}}-\mathrm{t}_{\mathrm{i}-1}\right)+\ln \left(\mathrm{c}_{\mathrm{p}_{\mathrm{i}} \mathrm{p}_{\mathrm{i}+1}}\right)\right)} \times\right.\right.$ $\left.\left.\times \frac{\mathrm{e}^{-\kappa_{\mathrm{p}_{\mathrm{n}+1}}\left(\mathrm{t}_{\mathrm{n}+1}-\mathrm{t}_{\mathrm{n}}\right)}-1}{-\kappa_{p_{n+1}}}\right)\right)$.

The above integral can be bounded by considering the cases $\kappa_{p}>0$ or $\kappa_{p}<0$ as follows

$\int_{0}^{\infty} P^{(s)}(\sigma) \rho_{p_{1}} d s \leq \frac{\rho_{\max }}{\kappa_{\min }}\left(\sum_{n=0}^{\infty}\left(\mathrm{e}^{\sum_{\mathrm{i}=0}^{\mathrm{n}}\left(-\kappa_{\mathrm{p}_{\mathrm{i}+1}}\left(\mathrm{t}_{\mathrm{i}+1}-\mathrm{t}_{\mathrm{i}}\right)+\ln \left(\mathrm{c}_{\mathrm{p}_{\mathrm{i}} \mathrm{p}_{\mathrm{i}+1}}\right)\right)}\right)\right.$,

where $\kappa_{\text {min }}:=\min _{p \in \mathcal{P}}\left|\kappa_{p}\right|$ and $c_{p_{0} p_{1}}=1$. Using the total running time of stable subsystems $T^{-}\left(t_{n}\right):=\bar{T}^{-}(n)$ and unstable subsystems $T^{+}\left(t_{n}\right):=\bar{T}^{+}(n)$, in $\left[0, t_{n}\right)$, (31) can be rewritten as

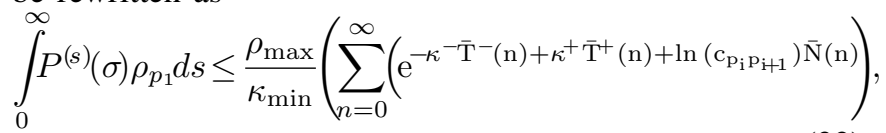

where $\kappa^{-}:=\min _{p \in\{1, \ldots, K\}} \kappa_{p}, \kappa^{+}:=\max _{p \in\{K+1, \ldots, M\}} \kappa_{p}$. Using (27) with (32), we obtain

$\int_{0}^{\infty} P^{(s)}(\sigma) \rho_{p_{1}} d s<\frac{\rho_{\max }}{\kappa_{\min }}\left(\sum_{n=0}^{\infty}\left(\mathrm{e}^{-\kappa^{*} \overline{\mathrm{T}}(\mathrm{n})+\ln \left(\mathrm{c}_{\mathrm{p}_{\mathrm{i}} \mathrm{p}_{\mathrm{i}+1}}\right) \overline{\mathrm{N}}(\mathrm{n})}\right)\right.$.

Note that (2) with $t=t_{n}$ follows that $\bar{T}(n) \geq\left(\bar{N}(n)-N^{0}\right) \tau$. Applying previous inequality to (33), we have

$$
\begin{aligned}
\int_{0}^{\infty} P^{(s)}(\sigma) \rho_{p_{1}} d s & <\frac{\rho_{\max } \mathrm{e}^{\kappa^{*} \tau \mathrm{N}_{0}}}{\kappa_{\min }}\left(\sum_{n=0}^{\infty}\left(\mathrm{e}^{\left(-\kappa^{*} \tau+\max _{\mathrm{p}, \mathrm{q} \in \mathcal{P}} \ln \left(\mathrm{c}_{\mathrm{pq}}\right)\right) \overline{\mathrm{N}}(\mathrm{n})}\right)\right. \\
& <\frac{\rho_{\max } \mathrm{e}^{\kappa^{*} \tau \mathrm{N}_{0}}}{\kappa_{\min }}\left(\sum_{n=0}^{\infty} \mathrm{e}^{\gamma \cdot \mathrm{n}}\right)
\end{aligned}
$$

where $\gamma:=-\kappa^{*} \tau+\max _{p, q \in \mathcal{P}} \ln \left(c_{p q}\right)$ and $\gamma<0$ due to (28). (34) implies that

$$
\int_{0}^{\infty} P^{(s)}(\sigma) \rho_{p_{1}} d s<\frac{\rho_{\max } \mathrm{e}^{\kappa^{*} \tau \mathrm{N}_{0}}}{\kappa_{\min }}\left(\sum_{n=0}^{\infty} \mathrm{e}^{\gamma \cdot \mathrm{n}}\right)=\frac{\rho_{\max } \mathrm{e}^{\kappa^{*} \tau \mathrm{N}_{0}}}{\kappa_{\min }\left(1-\mathrm{e}^{\gamma}\right)}<\infty .
$$

Thus, by means of Lemma 3, we can conclude that (1) with $\sigma \in \mathcal{S}_{A D T}[\tau]$ is almost globally stable if the conditions (27) and (28) are satisfied.

Remark 3: In Theorem 2, on contrary to the condition (13) in Theorem 1, we attain a multiple Lyapunov density to each unstable subsystem. Here, we aim to compensate the decrease of the density along the solutions of unstable subsystem with the growth of the density along the solutions of stable subsystems, the compatibility condition (26) and the ratio between the operation time of stable and unstable subsystems.

\section{AN EXAMPLE}

To show the applicability of the result, we provide an example of a switched system with stable and unstable subsystems. Consider (1) with the following subsystems

$$
\begin{aligned}
& f_{1}\left(x_{1}, x_{2}\right)=\left(\begin{array}{c}
0.2 x_{1}+\frac{4}{3} x_{2}+3 x_{1}^{2}-16 x_{2}^{2} \\
-0.75 x_{1}+0.2 x_{2}+12 x_{1} x_{2}
\end{array}\right), \\
& f_{2}\left(x_{1}, x_{2}\right)=\left(\begin{array}{c}
-0.6 x_{1}-1.4 x_{2}+28 x_{1} x_{2} \\
\frac{5}{7} x_{1}-0.6 x_{2}-\frac{75}{7} x_{1}^{2}+7 x_{2}^{2}
\end{array}\right), \\
& f_{3}\left(x_{1}, x_{2}\right)=\left(\begin{array}{c}
-0.4 x_{1}+\frac{5}{6} x_{2}+6 x_{1}^{2}-12.5 x_{2}^{2} \\
-1.2 x_{1}-0.4 x_{2}+24 x_{1} x_{2}
\end{array}\right) .
\end{aligned}
$$

The densities corresponding to subsystems are given as $\rho\left(x_{1}, x_{2}\right)=\rho_{1}\left(x_{1}, x_{2}\right)=\left(\left(3 x_{1}\right)^{2}+\left(4 x_{2}\right)^{2}\right)^{-3}, \rho_{2}\left(x_{1}, x_{2}\right)=$ $\left(\left(5 x_{1}\right)^{2}+\left(7 x_{2}^{2}\right)\right)^{-3}$, and $\rho_{3}\left(x_{1}, x_{2}\right)=\left(\left(6 x_{1}\right)^{2}+\left(5 x_{2}^{2}\right)\right)^{-3}$. Condition (11) is valid since $\left(1+\left\|f_{1}\right\|\right) \rho \leq L\|x\|^{-4}$, where $L>0$ and $\left(1+\left\|f_{p}\right\|\right) \rho_{p} \leq L_{p}\|x\|^{-4}$, where $L_{p}>0$, $p=2$, 3. Moreover, (12) and (13) are valid since $\nabla \cdot\left(f_{p} \rho_{p}\right)=$ $\kappa_{p} \rho_{p}, \kappa_{1}=\kappa=-0.8, \kappa_{2}=2.4$ and $\kappa_{3}=1.6$. By means of $c=(4 / 5)^{6}, c_{2}=(7 / 3)^{6}, c_{3}=2^{6}$, we satisfy (14)-(16). Since the conditions of Theorem 1 are satisfied, we can say (1) with (36) and $\mathcal{S}=\mathcal{S}_{M D A D T}\left[\tau^{f}\right] \cup \mathcal{S}_{M D A D T}\left[\left\{\tau_{2}, \tau_{3}\right\}\right]$ is almost globally stable if $\tau^{f}<1.67, \tau_{2}>2.12$ and $\tau_{3}>2.60$. The bounds for the mode-dependent dwell times are obtained via (3) and (4).

Periodic switching signals are used in the figures and they are described by writing the shortest repeating pattern as 
$\sigma=\left\{\left(\Delta t_{1}, p_{1}\right),\left(\Delta t_{2}, p_{2}\right), \ldots,\left(\Delta t_{n}, p_{n}\right),\left(\Delta t_{1}, p_{1}\right), \ldots\right\}=$ $\left\{\left(\Delta t_{1}, p_{1}\right),\left(\Delta t_{2}, p_{2}\right), \ldots,\left(\Delta t_{n}, p_{n}\right)\right\}$.

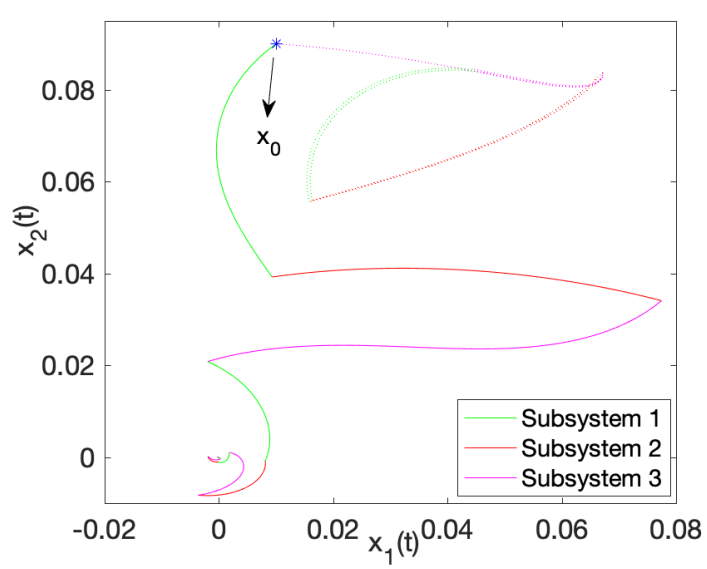

Fig. 1. The dotted curve is drawn for a backward solution of (1) with (36) and with a periodic switching signal $\{(2.15,2),(1.65,1),(2.67,3)\}$. As $t \rightarrow-\infty$, the solution approaches to a limit cycle. The plain curve is drawn for the forward solution with the same switching signal and as $t \rightarrow \infty$, the solution approaches to origin.

In Figure 1, a forward and a backward solution of (1) with (36) and with a periodic switching signal $\{(2.15,2),(1.65,1),(2.67,3)\}$ are drawn. The forward solution approaches to origin as $t \rightarrow \infty$ and the backward solution approaches to a limit cycle as $t \rightarrow-\infty$. It implies that some solutions (included in a set with Lebesgue measure zero) may not be attracted by the origin as $t \rightarrow \infty$.

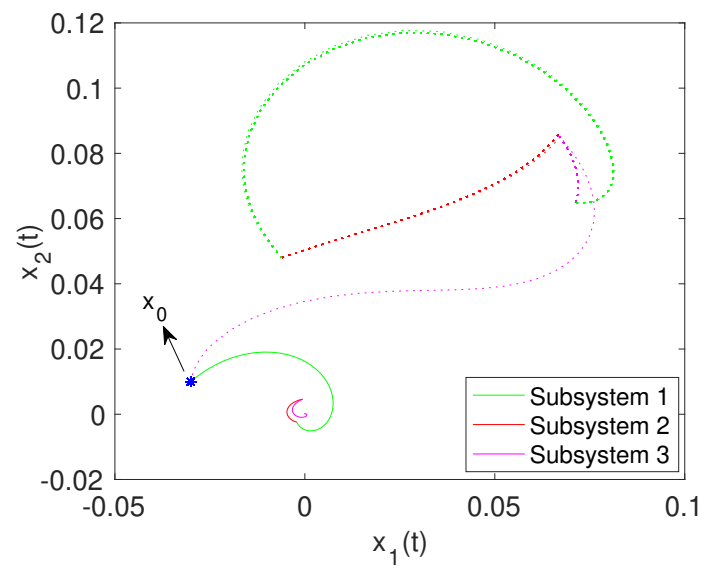

Fig. 2. The plain curve is drawn for the forward solution of (1) with (36) and with a periodic switching signal $\{(5.8,2),(2.5,1),(7.2,3)\}$. The dotted curve is drawn for backward solution with the same initial data and the same signal. The signal has average dwell time approximately 5.17 and $\frac{T^{-}(t)}{T^{+}(t)} \approx 5.2$. As $t \rightarrow \infty$, the solution approaches to origin. As $t \rightarrow-\infty$, solution starting from $x_{0}$ approaches to a limit cycle.

Conditions (12), (24) and (25) of Theorem 2 have already been showed to be satisfied above and (26) can be satisfied with $c_{12}=(7 / 3)^{6}, c_{21}=(4 / 5)^{6}, c_{13}=2^{6}, c_{31}=(4 / 5)^{6}$, $c_{23}=(6 / 5)^{6}$ and $c_{32}=(7 / 5)^{6}$. Taking $\kappa^{*}=1 \in(0,1.6)$, we obtain that $\tau>\ln \left((7 / 3)^{6}\right) \approx 5$.08. From Theorem 2 , (1) with (36) is almost globally stable for any switching signal with average dwell time greater than 5.08 and $\frac{T^{-}(t)}{T^{+}(t)}>3$. In Figure 2, the forward and backward solutions of (1) with (36) and with the given periodic switching signal are depicted. The forward solution approaches to the origin as time increases to $\infty$. Likewise, the backward solution approaches to a limit cycle as time decreases to $-\infty$.

\section{CONCLUSION}

We have provided two sufficient conditions to ensure almost global stability of switched systems. The first one is based on fast switching of unstable subsystems and slow switching of stable subsystems. Whereas, the second one is based on slow switching of all systems with the rate, where the ratio of operation times of stable and unstable subsystems in a certain range. We have obtained lower and upper bounds for mode-dependent average dwell time for stable subsystems and unstable subsystems, respectively. With the help of the ratio between operation times of stable and unstable subsystems, a lower bound for average dwell time has been derived.

\section{REFERENCES}

[1] D. Liberzon, Switching in systems and control. Springer Science \& Business Media, 2003.

[2] J. Geromel and P. Colaneri, "Stability and Stabilization of ContinuousTime Switched Linear Systems," SIAM Journal on Control and Optimization, vol. 45, no. 5, pp. 1915-1930, 2006.

[3] J. P. Hespanha and A. S. Morse, "Stability of switched systems with average dwell-time," in Proceedings of the 38th IEEE Conference on Decision and Control, vol. 3, Dec 1999, pp. 2655-2660 vol.3.

[4] X. Zhao, L. Zhang, P. Shi, and M. Liu, "Stability and stabilization of switched linear systems with mode-dependent average dwell time," IEEE Transactions on Automatic Control, vol. 57, no. 7, pp. 18091815 , July 2012.

[5] J. Yang, X. Zhao, X. Bu, and W. Qian, "Stabilization of switched linear systems via admissible edge-dependent switching signals," Nonlinear Analysis: Hybrid Systems, vol. 29, pp. 100 - 109, 2018.

[6] G. Zhai, B. Hu, K. Yasuda, and A. N. Michel, "Stability analysis of switched systems with stable and unstable subsystems: An average dwell time approach," International Journal of Systems Science, vol. 32 , no. 8, pp. 1055-1061, 2001.

[7] D. Xie, H. Zhang, H. Zhang, and B. Wang, "Exponential stability of switched systems with unstable subsystems: A mode-dependent average dwell time approach," Circuits, Systems, and Signal Processing, vol. 32, no. 6, pp. 3093-3105, Dec 2013.

[8] Y. Wang, H. R. Karimi, and D. Wu, "Conditions for the stability of switched systems containing unstable subsystems," IEEE Transactions on Circuits and Systems II: Express Briefs, vol. 66, no. 4, pp. 617-621, April 2019.

[9] A. Rantzer, "A dual to Lyapunov's stability theorem," Systems \& Control Letters, vol. 42, pp. 1-17, 2001.

[10] I. Masubuchi and T. Kikuchi, "Lyapunov density for almost attraction of nonlinear time-varying systems: A condition without assuming local stability," in 2017 25th Mediterranean Conference on Control and Automation (MED), July 2017, pp. 169-173.

[11] I. Masubuchi and Y. Ohta, "Analysis of almost-everywhere stability of a class of discontinuous systems via Lyapunov densities," in 2016 European Control Conference (ECC), June 2016, pp. 567-574.

[12] R. Rajaram and U. Vaidya, "Lyapunov density for coupled systems," Applicable Analysis, vol. 94, no. 1, pp. 169-183, 2015.

[13] Ö. Karabacak, A. Kıvılcım, and R. Wisniewski, "Almost global stability of nonlinear switched systems with time-dependent switching," IEEE Transactions on Automatic Control, vol. 65, no. 7, pp. 29692978, 2020.

[14] F. İlhan, Ö. Karabacak, and R. Wisniewski, "A sufficient condition for the almost global stability of nonlinear switched systems with average dwell time," in 2019 IEEE 58th Conference on Decision and Control (CDC), 2019, pp. 8013-8017.

[15] A. Lasota and M. C. Mackey, Chaos, fractals and noise: Stochastic aspects of dynamics. New York: Springer-Verlag, 1994.

[16] Ö. Karabacak, R. Wisniewski, and J. Leth, "On the almost global stability of invariant sets," in 2018 European Control Conference (ECC), June 2018, pp. 1648-1653. 